\title{
Declassified Intelligence Satellite Photographs
}

Declassified photographs from U.S. intelligence satellites provide an important worldwide addition to the public record of the Earth's land surface. This imagery was released to the National Archives and Records Administration (NARA) and the U.S. Geological Survey (USGS) in accordance with Executive Order 12951 on February 23, 1995. The NARA has the original declassified film and a viewing copy. The USGS has another copy of the film to complement the Landsat archive.

The declassified collection involves more than 990,000 photographs taken from 1959 through 1980 and was released on two separate occasions: February 1995 (Declass 1) and September 2002 (Declass 2). The USGS copy is maintained by the Earth Resources Observation and Science (EROS) Center, near Sioux Falls, South Dakota. Both the NARA and EROS provide public access to this unique collection that extends the record of landsurface change back another decade from the advent of the Landsat program that began satellite operations in 1972.
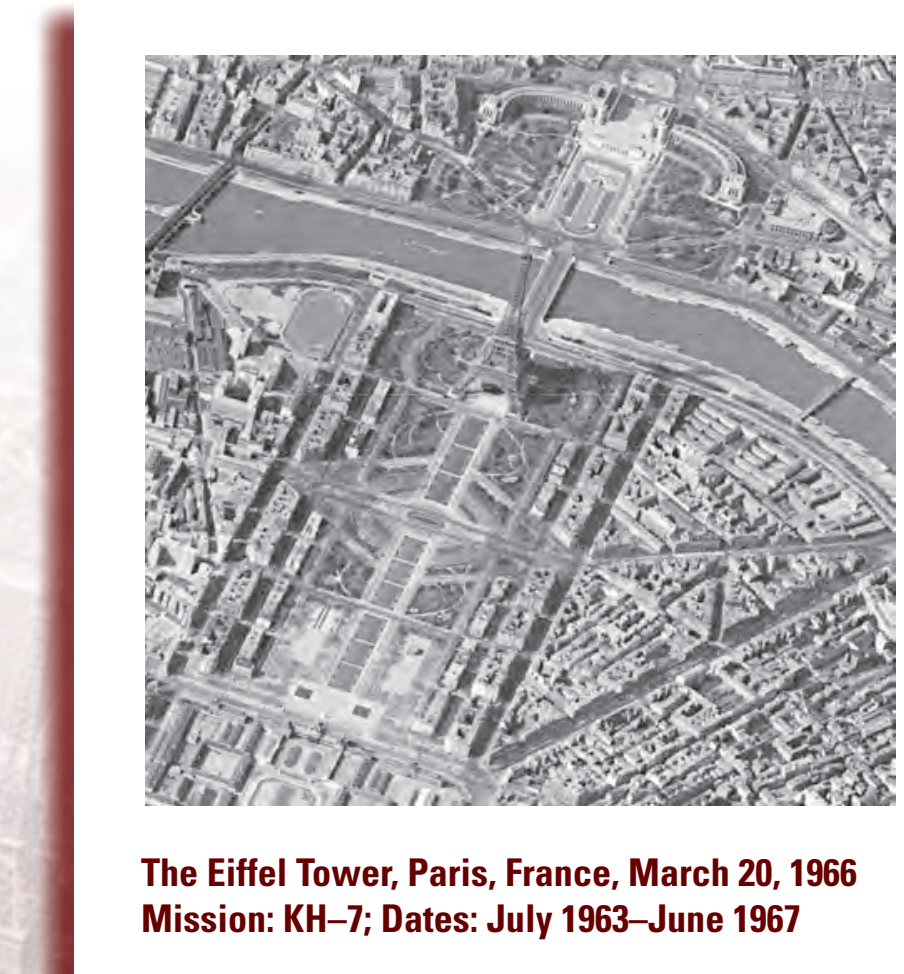
The Eiffel Tower, Paris, France, March 20, 1966 Mission: KH-7; Dates: July 1963-June 1967 (Mission: KH-7: Dates: July $1963-J u n e$ 1967

\section{Summary of Satellite Missions}

KH-1 Dates: June 1959-Sept. 1960

Ground Resolution: 25 feet

Notes: Single camera

Frames: 1,432

Satellite System: CORONA

Mission Number: 9009

KH-2 Dates: Oct. 1960-Oct. 1961

Ground Resolution: 25 feet

Notes: Single camera

Frames: 7,246

Satellite System: CORONA

Mission Numbers: 9013, 9017, 9019

KH-3 Dates: Aug. 1961-Jan. 1962

Ground Resolution: 25 feet

Notes: Single camera

Frames: 9,918

Satellite System: CORONA

Mission Numbers: 9022, 9023, 9025, 9028, 9029 


\section{Summary of Satellite Missions-Continued}

KH-4B Dates: Sept. 1967-May 1972

Ground Resolution: 6 feet

Notes: Two cameras

Frames: 188,526

Satellite System: CORONA

Mission Numbers: 1101 through 1112, 1114, 1006 through 1117

KH-5 Dates: Feb. 1961-Aug. 1964

Ground Resolution: 460 feet

Notes: Global coverage,

mapping camera

Frames: 38,578

Satellite System: ARGON

Mission Numbers: 9034A, 9046A, 9058A, 9059A, 9066A

KH-6 Dates: July 1963

Ground Resolution: 6 feet

Notes: Panoramic camera

Frames: less than 910

Satellite System: LANYARD

Mission Numbers: 8003

KH-7 Dates: July 1963-June 1967

Ground Resolution: 2 feet

Frames: 18,000

Mission Numbers: 4001 through 4038

KH-9 Dates: Mar. 1973-Oct. 1980

Ground Resolution: 20 feet

Frames: 29,000

Mission Numbers: $1205-5$ through 1216-5

\section{Available Products}

To search our archives, please visit EarthExplorer:

http://earthexplorer.usgs.gov

\section{For More Information}

U.S. Geological Survey

Earth Resources Observation and Science (EROS) Center

47914 252nd Street

Sioux Falls, SD 57198-0001

E-mail: 1ta@usgs.gov 